\title{
Nanoalloying in real time. A high resolution STEM and computer simulation study $\dagger$
}

\author{
M. M. Mariscal, ${ }^{* a}$ A. Mayoral, ${ }^{b}$ J. A. Olmos-Asar, ${ }^{a}$ C. Magen, ${ }^{b c d}$ S. Mejía-Rosales, ${ }^{e}$ E. Pérez-Tijerina ${ }^{e}$ \\ and M. José-Yacamán $f$
}

Received 9th August 2011, Accepted 27th September 2011

DOI: $10.1039 / \mathrm{c} 1 \mathrm{nr} 11052 \mathrm{~g}$

Bimetallic nanoparticles constitute a promising type of catalysts, mainly because their physical and chemical properties may be tuned by varying their chemical composition, atomic ordering, and size. Today, the design of novel nanocatalysts is possible through a combination of virtual lab simulations on massive parallel computing and modern electron microscopy with picometre resolution on one hand, and the capability of chemical analysis at the atomic scale on the other. In this work we show how the combination of theoretical calculations and characterization can solve some of the paradoxes reported about nanocatalysts: Au-Pd bimetallic nanoparticles. In particular, we demonstrate the key role played by adsorbates, such as carbon monoxide (CO), on the structure of nanoalloys. Our results imply that surface condition of nanoparticles during synthesis is a parameter of paramount importance.

\section{Introduction}

The boom in nanotechnology research and development of new methods in the last few decades has helped to begin to understand the mechanisms that control the growth of bimetallic particles. This has been a major technological problem affecting a variety of applications ranging from environment to new energy sources. For instance, a very significant application is the case of fuel cells which are severely limited to the use of platinum in large concentrations. However, since this metal is very scarce,

${ }^{a}$ INFIQCICONICET, Departamento de Matemática y Física, Facultad de Ciencias Químicas, Universidad Nacional de Córdoba, Córdoba, Argentina.E-mail: marcelo.mariscal@conicet.gov.ar

${ }^{b}$ Laboratorio de Microscopías Avanzadas (LMA), Instituto de Nanociencia de Aragón (INA), Universidad de Zaragoza, 50018 Zaragoza, Spain

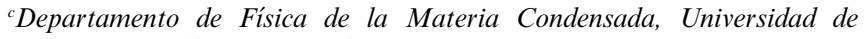
Zaragoza, 50009 Zaragoza, Spain

${ }^{d}$ Fundación ARAID, 50004 Zaragoza, Spain

${ }^{e}$ Laboratorio de Nanociencias y Nanotecnología, Facultad de Ciencias Físico-Matemáticas, Universidad Autónoma de Nuevo León, San Nicolás de los Garza, Nuevo León, 66450, México

${ }^{f}$ Department of Physics and Astronomy, The University of Texas at San Antonio, One UTSA Circle, 78251 San Antonio, Texas, USA

$\dagger$ Electronic supplementary information (ESI) available: (S1) (a) Aberration corrected STEM-HAADF image taken after beam irradiation, where the intensity profile, marked by a red rectangle, was taken. The white arrow indicates the analysis direction. (S2) Elemental analysis carried out on a nanoparticle composed of $\mathrm{Au}$ and Pd. (a) STEM-HAADF image with the line where the scan was performed pointing to the scan direction and (b) line profile results where the blue color corresponds to $\mathrm{Au}$ and red color to Pd. Table S1: adsorption energies $E_{\text {ads }}$ and equilibrium bond distances $r_{\mathrm{e}}$ obtained from the DFT calculations of $\mathrm{CO}$ adsorption on $\mathrm{Pd}$ clusters. The respective bond-orders BO and the fitted Morse potential parameter $\left(D_{\mathrm{e}}\right)$ are given in the last columns. See DOI: 10.1039/c1nr11052g a massive application using large amounts of it would be physically impossible. Therefore, one of the most important challenges for the commercialization of fuel cells is the preparation of robust and low-cost catalysts. In the last years a great emphasis has been placed on the design of bimetallic nanoparticles to improve the performance of many catalysts because their unique catalytic properties are often superior to those of pure metals. ${ }^{1}$

We can speculate that if enough control of nanoparticle growth is achieved, nanoparticles with a core of a less scarce metal (such as copper) and an external layer made of one monolayer of platinum could be designed.

A fundamental goal of nanocatalysis research is to produce catalysts with maximum selectivity, extremely high activity, low energy consumption, low production cost and long lifetime. This can only be achieved by precisely controlling the size, shape, chemical distribution and surface composition of nanoparticles.

For a very small size - just a few nanometres - reactivity of metals can dramatically increase; as a consequence, metal nanoparticles (NPs) can exhibit a variety of catalytic properties with many practical applications. A good example is gold, which goes from being a non-reactive "noble" metal in bulk form to becoming a good catalyst when it is forming small clusters of few atoms. ${ }^{2-4}$ It has also been shown that Pd NPs are more active than $\mathrm{Pd}$ black and $\mathrm{Pd} / \mathrm{Al}_{2} \mathrm{O}_{3}$ powders. ${ }^{5}$ The higher activity could be understood in terms of the high surface to volume ratio and the great amount of low-coordinated sites on the surface produced by surface dislocations or disorder, which can make the particles very reactive. The combination of $\mathrm{Au}$ and $\mathrm{Pd}$ to form a bimetallic catalyst has been studied for a number of reactions: aromatics hydrogenation, ${ }^{5}$ vinyl acetate synthesis, ${ }^{4}$ trichloroethene hydrodechlorination- $\mathrm{TCE}^{6}$ (the most common hazardous contaminants in groundwater), gas-phase 
hydrodehalogenation of fluorinated compounds, ${ }^{7}$ hydrodesulfurization reactions, ${ }^{8}$ direct oxidation of hydrogen to form hydrogen peroxide ${ }^{9}$ or $n$-heptane isomerization, ${ }^{10}$ cyclotrimerization of acetylene, selective oxidation of alcohols, and in applications such as hydrogen fuel cells and pollution control systems. ${ }^{11,12}$

Accordingly, the design of efficient catalysts based on metal nanostructures, a fair understanding of the role of structure, elemental composition, size, and morphology have to be achieved, and in order to reach this level of knowledge, the ability to produce metal nanoparticles with a good control of these features is critical. Chemical methods can produce nanoparticles with controlled shape and size but the kinetic processes involved in the method make it rather difficult to control the appearance of core-shell structures..$^{13}$ On the other hand, physical methods such as inert gas condensation have emerged as a very good technique. This allows a narrower size distribution and a great control on the elemental composition of the nanoparticles, as it mainly depends on the composition of the target. ${ }^{14-16}$ Through this production method, particles with sizes below $5 \mathrm{~nm}$ adopting decahedral or icosahedral morphologies can be easily obtained. However, the understanding of the specific processes involved in the production of the particles is incomplete, and this lack of knowledge limits the capacity of avoiding unwanted issues, such as agglomeration, that can be crucial in the lifetime of the catalyst. ${ }^{17,18}$

Asoro et al. ${ }^{19}$ have monitored the coalescence of Pt nanoparticles by aberration $\left(C_{\mathrm{s}}\right)$ corrected STEM, and they have demonstrated a methodology for determining fundamental sintering parameters, such as mass transport and grain boundary mobility.

From a theoretical point of view, the energy, structure and segregation (chemical ordering) of $\mathrm{Pd}-\mathrm{Au}$ nanoalloys have been reported, using a genetic algorithm ${ }^{20,21}$ for global geometry optimization. In these works, the binding energy and the second difference in energy were used as stability criteria. Using different parameterizations of the Gupta semiempirical potential they found $\mathrm{Pd}_{\text {core }} \mathrm{Au}_{\text {shell }}$ segregation, and $\mathrm{Pd}-\mathrm{Au}$ mixing. Very recently, using density functional calculations (DFT), Yudanov and Neyman, ${ }^{22}$ reported that segregation of $\mathrm{Au}$ atoms to the edges of the particle surface is thermodynamically favorable, whereas $\mathrm{Pd}$ occupies the facets. Therefore, the formation of $\mathrm{Au}_{\text {core }} \mathrm{Pd}_{\text {shell }}$ nanoalloys seems to be unfavorable considering only bare metallic nanoparticles. $\mathrm{Pd}_{\text {core }} \mathrm{Au}_{\text {shell }}$ segregation is favored by the lower surface energy of $\mathrm{Au}\left(96.8 \mathrm{meV}^{\circ} \AA^{-2}\right)$ against $\operatorname{Pd}\left(131 \mathrm{meV}^{-2}\right)$ which minimizes the overall nanoparticle surface energy.

This work solves some of the paradoxes reported about $\mathrm{Au}-\mathrm{Pd}$ bimetallic nanoparticles, in particular the structure and chemical ordering of one of the most important nanoalloys for catalysis and a precursor for the growth of metal nanoparticles for cancer research and treatment. ${ }^{4,23-25}$

This paper aims to contribute to a better understanding of nanoalloys forming mechanisms, focusing specifically on $\mathrm{Au} / \mathrm{Pd}$ nanoparticles, one of the most important nanoalloys for catalysis. The core of this study lies in the use of computer simulations in combination with aberration corrected STEM. We use a high angle annular dark field (HAADF) detector to investigate the coalescence of the nanoalloys when the microgrids where the particles are deposited are irradiated by the electron beam. The use of the inert gas condensation technique is appropriate because in this kind of synthesis it is possible to distinguish the original particles from the coalesced particles by measuring their sizes, while the use of high resolution HAADF microscopy allows one to make an in-depth study on how the electron beam affects the morphology, elemental distribution, and structure of the nanoparticles.

\section{Methods}

\section{Synthesis of NPs}

Metal nanoparticles and nanoalloys were produced with a sputtering system Nanogen 50 from Mantis Deposition Ltd using what is known as Inert Gas Condensation (IGC) method. ${ }^{26,27}$ In the IGC process, a supersaturated vapor of metal atoms is originated by sputtering a metal target in an inert gas atmosphere of $\mathrm{Ar}$ and $\mathrm{He}$. The Nanogen 50 system was kept at low temperature by a coolant mixture, and before the deposition of the nanoparticles the system pressure was set at $1 \times$ $10^{-9}$ Torr.

Under these conditions, gold and palladium nanoparticles were produced in a two-step fashion using inert gas condensation. Firstly, a target composed of the metal A (either Au or Pd) was used to grow monometallic nanoparticles, which - after their flight into the synthesis chamber-were deposited onto a substrate. Subsequently, the procedure is repeated on material $\mathrm{B}$ (either Pd or $\mathrm{Au}$ ), making the deposition on the same substrate used with metal A. In the case of nanoalloys, an alloyed target was employed to produce the particles in a single step procedure.

The production rate and size of the nanoparticles were controlled through the variation of gas flow, partial pressure, magnetron power, and condensation zone length. These parameters were optimized to produce particles of $5.0 \mathrm{~nm}$ in diameter. The nanoparticles were deposited directly onto the carbon copper microgrids, which were used for observation inside the electron microscope.

\section{Characterization}

The materials obtained were characterized using a JEOL JEMARM200F operated at $200 \mathrm{kV}$ (point resolution $0.8 \AA$ ) and a high brightness gun (X-FEG) FEI TITAN 60-300 operated at $300 \mathrm{kV}$ (point resolution $0.8 \AA$ ) equipped with a monochromator and a Gatan Tridiem 866 ERS energy filter (EELS/ EFTEM) for spectroscopy analysis. STEM images were simultaneously recorded in bright field (BF) and HAADF modes. In both microscopes, the probe correction was performed through a dodecapole corrector (CEOS $\mathrm{GmbH}$ ) aligned through the CESCOR software. The HAADF images were obtained setting the annular detector to make sure that it easily satisfied the requirement to eliminate contributions from unscattered or low-angle scattered electrons. In both cases, the pixel spacing was calibrated using $\mathrm{Si} 110$ lattice images in HAADF mode; it was confirmed by a standard sample composed of gold particles on amorphous carbon. Images were commonly recorded from 10 to 16 seconds. The scanning size of the EELS map was $43 \times 56$ pixels using an acquisition time of 0.12 seconds. 


\section{Computer simulation details}

We have performed energy minimizations using a simulated annealing procedure to find relaxed structures. A temperature program starting from $50 \mathrm{~K}$ which was then increased linearly until $400 \mathrm{~K}$ was used. Finally, temperature was reduced exponentially until it reached $0.5 \mathrm{~K}$, using the Andersen thermostat with a velocity-Verlet algorithm to produce the trajectories. We have used a new interatomic potential, recently developed by Mariscal et al. ${ }^{28,29}$ to represent the interaction between various atoms. The force-field is based on a bond-order Morse potential to represent the interaction between $\mathrm{CO}$ molecules and the $\mathrm{Pd} /$ $\mathrm{Au}$ atoms. To generate the required parameters, density functional calculations (DFT) were required as stated in our previous work. $^{29}$

The DFT calculations were performed using a cluster of $25 \mathrm{Pd}$ atoms with a CO molecule adsorbed on different sites (see ESI, Table S1 $\dagger$ ). In each of the configurations selected, with the molecule adsorbed on the equilibrium position (i.e., CO-Pd distance at the $r_{\mathrm{e}}$ value), $D_{\mathrm{e}}$ parameter was changed self-consistently, keeping $\alpha$ as a constant, until the adsorption energy value predicted by DFT calculations was reproduced. The optimized values are shown in the ESI (Table $\mathrm{S} 1 \dagger$ ). The calculations within the framework of DFT were performed with the SIESTA code. ${ }^{30}$ The valence electrons were described with a set of double-z polarized (DZP) bases. The exchange and correlation effects were described using the generalized gradient approximation (GGA) in the functional Perdew-Burke-Ernzerhof (PBE). Results obtained, together with the potential parameters $D_{\mathrm{e}}$ and $r_{\mathrm{e}}$, are listed in Table S1 (see ESI $\dagger$ ).

Adsorption of $\mathrm{CO}$ on the $\mathrm{Au}$ surface shows slightly positive values or close to zero; therefore we set the $D_{\mathrm{e}}$ parameter equal to zero for all sites to simplify the calculations. This is in accordance with a recent study reported by Abbott et al. ${ }^{31}$

Metallic interactions were modeled using the second-moment approximation of the tight binding theory ${ }^{32}$ (TB-SMA) which takes into account the many-body character of the metallic bond and has been proved to reproduce most of the characteristic properties of gold and palladium. We chose the parameter set III reported by Ismail and Johnston ${ }^{20}$ Molecular dynamics simulations using our homemade code were also performed to simulate the coalescence process between $\mathrm{Pd}$ and $\mathrm{Au}$ nanoparticles of 309 atoms (approximately $2 \mathrm{~nm}$ of diameter). The velocity-Verlet algorithm was used to integrate the equation of motions with an integration step of $1 \mathrm{fs}$. All coalescence simulations were performed in the canonical ensemble (NVT) using the Andersen thermostat to keep the temperature constant at $500 \mathrm{~K}$.

\section{Results and discussions}

We show the synthesis of $\mathrm{Au} / \mathrm{Pd}$ nanoparticles by inert gas condensation and their characterization by aberration-corrected scanning transmission electron microscopy (STEM), using a high angle annular dark field (HAADF) detector to investigate nanoalloying in real time. The STEM study shows in atomicdetail the rearrangement of Pd under the effect of the electron beam, and the differences originated by the two methods of deposition. Using computer simulations we propose that $\mathrm{CO}$ adsorption on $\mathrm{Pd}$ nanoparticles is critical to obtain the
$\mathrm{Au}_{\text {core }}-\mathrm{Pd}_{\text {shell }}$ structures observed with STEM, instead of $\mathrm{Pd}_{\text {core }}{ }^{-}$ $\mathrm{Au}_{\text {shell }}$ as theoretically predicted by previous studies.

The nanoalloying mechanism of $\mathrm{Au}-\mathrm{Pd}$ NPs was investigated in real time by atomic resolution imaging of the coalescence of $\mathrm{Au}$ and $\mathrm{Pd}$ during the electron beam irradiation inside a transmission electron microscope. The processes were monitored by Cs STEM-HAADF microscopy coupled to electron energy loss spectroscopy (EELS). In HRTEM the contrast is strongly related to the atomic weight of the element, and the location of $\mathrm{Au}$ and $\mathrm{Pd}$ atoms can be easily tracked.

Gold and palladium nanoparticles with average size of $5 \mathrm{~nm}$ were grown by inert gas deposition onto holey carbon copper microgrids. The materials were named $\mathrm{X} / \mathrm{Y}$, where " $\mathrm{X}$ " was the first metal deposited onto the grid. When the target employed was already an alloy it was named X@Y. In the present study we have focused our interest on the same system through three different approaches: $\mathrm{Au} / \mathrm{Pd} ; \mathrm{Pd} / \mathrm{Au}$ and $\mathrm{Au} @ \mathrm{Pd}$ in order to avoid any effect of the synthesis conditions.

\section{Au/Pd and Pd/Au}

For these two experiments, $\mathrm{Au}$ and $\mathrm{Pd}$ - and vice versa-nanoparticles of approximately $5 \mathrm{~nm}$ in diameter were deposited separately onto the microgrid. Fig. 1a shows a scheme of the system, where some metal nanoparticles of two different chemical natures were deposited onto amorphous carbon. Fig. 1b displays an aberration corrected STEM-HAADF image of the as-synthesized products. The image is formed by incoherent electrons scattered at high angles, and the intensity depends on the atomic number $(Z)$. Therefore, bright particles correspond to gold $(Z=79)$ and dark particles are $\operatorname{Pd}(Z=46)$. The order in which metals were deposited did not produce significant differences. After recording the initial data, the sample was kept under the electron beam irradiation over several minutes in order to force the mobility of the nanoparticles. Fig. 1c-f show a series of aberration corrected images of gold and palladium NPs which are evolving with time. For Fig. 1c-f the electron beam of $2.8 \mathrm{pA}$ was focused onto a small area of about $12 \mathrm{~nm}^{2}$ and kept constant for several minutes. The original size of the Pd nanoparticle was $6 \mathrm{~nm}$ in diameter and it presented a structured core (internal dotted circle, Fig. 1c) surrounded by a disordered shell (external dashed circle, Fig. 1c). The much brighter gold nanoparticle exhibited good crystallinity, adopting a decahedral shape oriented along its [111] direction with a diameter of $4.7 \mathrm{~nm}$. Both crystals were initially linked through a neck of $3.6 \mathrm{~nm}$. Fig. 1d presents the images recorded after two minutes when no big changes had taken place, except for a minimum tilting produced by irradiation when placing Au nanoparticles right on the axis with respect to the electron beam. Moreover, a darker contrast can be observed in both figures (white arrow), which was not present in Fig. 1c. This change is attributed to the presence of $\mathrm{Pd}$ coating the $\mathrm{Au}$, which became larger after 5 and 10 minutes (Fig. 1e and f). During the entire coalescence process, it was observed that the gold nanoparticle remained stable in its original position on the grid. The $\mathrm{Pd}$ diffused over the carbon to coalesce with the $\mathrm{Au}$ through the initial formation of a "neck", coating the gold and forming a core-shell structure $\left(\mathrm{Au}_{\text {core }} \mathrm{Pd}_{\text {shell }}\right)$. Further evidence of the process of coalescence was the size change occurred in the Pd NP which decreased from 

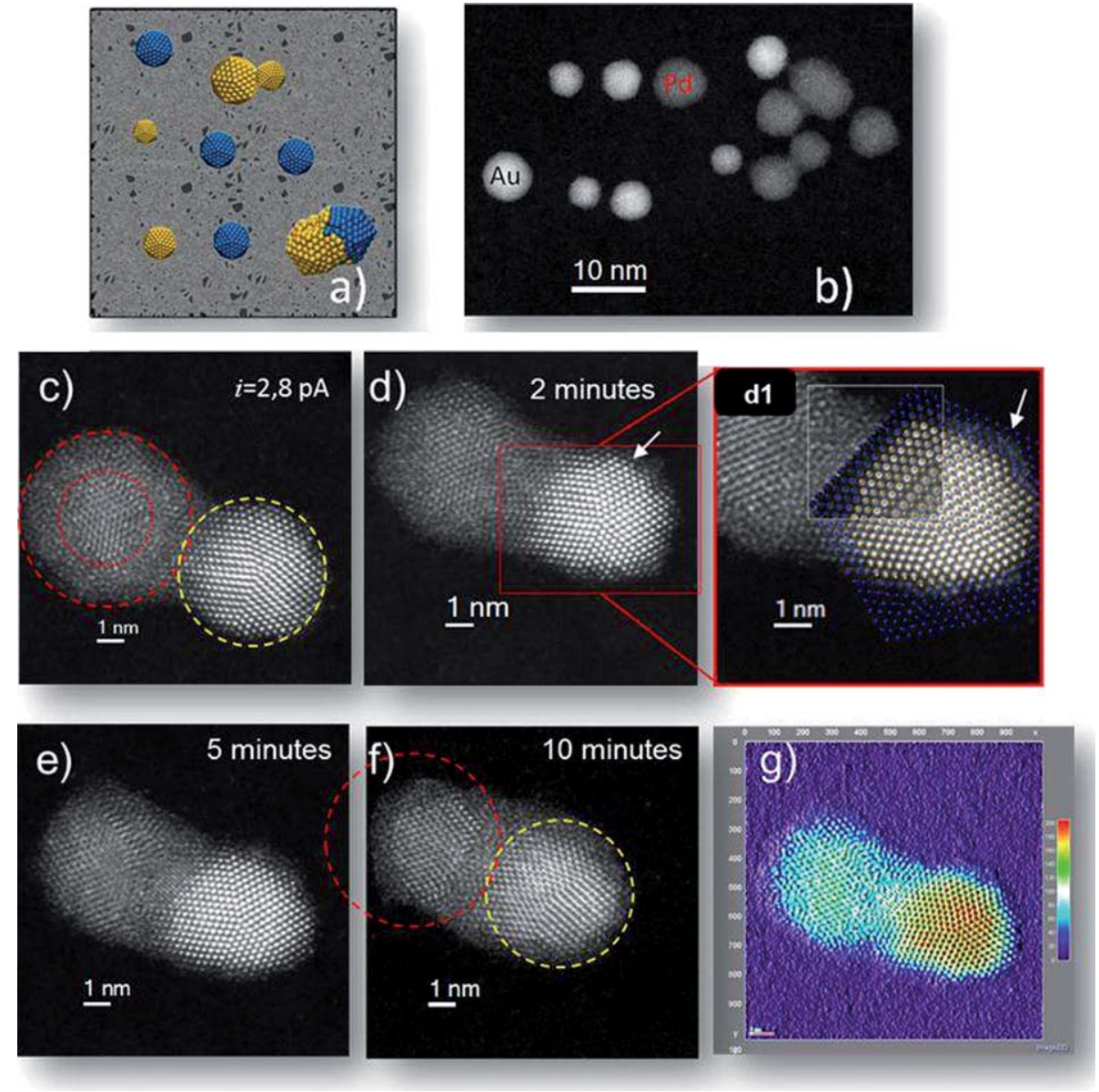

Fig. 1 (a) Schematic model representing the supported nanoparticles, (b) low magnification spherical-aberration (Cs) corrected STEM-HAADF images of Au (bright) and Pd (dark) nanoparticles. (c-f) Sequence of Cs-STEM-HAADF images recorded over several minutes at an electron beam of $2.8 \mathrm{pA}$. (d1) Comparison of the real micrograph shown at (d), with a simulated micrograph (small squared region) of a decahedral AuPd particle. The elemental distribution of the model particle is represented by yellow (gold) and blue (palladium) spheres. The area in the simulated STEM image that does not correspond to the decahedron was made transparent for clarity. (g) Artificially colored intensity map profile.

$6 \mathrm{~nm}$ to $4.8 \mathrm{~nm}$ (see the size of the external dashed circle for reference in Fig. 1f). An artificially colored map is plotted in Fig. 1g. This map was created based on the intensity measurements of each atom and it more clearly shows the difference in contrast between $\mathrm{Au}$ and $\mathrm{Pd}$.

By plotting the intensity profile over crystals after beam irradiation, the formation of a core-shell structure can be confirmed (see ESI, S1 $\dagger$ ). In addition, two similar nanoparticles, $\mathrm{Pd}$ and $\mathrm{Au}$, were analyzed by means of energy-dispersive X-ray spectroscopy (EDX), ESI S2†, to unambiguously corroborate the relation between composition and intensity. An EDX line profile was performed rastering the icosahedral $\mathrm{Au} \mathrm{NP}$ and the ordered Pd NP confirming their composition.

One of the main sources of interest in using high resolution STEM, instead of similar electron microscopy techniques, is the direct dependency of the intensity signal on the atomic number of the atoms present in the specimen. This, and the fact that in STEM the defocus value does not have an effect on intensity as strong as on conventional TEM, make this technique very convenient for the study of metallic systems. The intensity signal generated by an atom in STEM goes approximately as $Z^{1.5}$, and thus the contrast between gold and palladium should be evident.
But, since also the length of a particular atomic column will affect the intensity of the spot that represents that column in the micrograph, a model for the geometry and orientation of the particle is necessary to make a fair interpretation of the STEM image. So we generated such a model for the micrograph, which is shown in Fig. 1d. A decahedron was used to describe the geometry of the five-fold particle (even when the particle is not strictly decahedral on its borders, it is close enough to a decahedron at the region where $\mathrm{Au}$ and Pd meet), and the chemical composition was defined following the intensity differences in the micrograph. This model was used to generate a simulated STEM micrograph; the STEM simulations were made using the xHREM suite, ${ }^{33}$ based on the Ishizuka algorithm of the FFT multislice method. ${ }^{34}$ The simulated electron beam was of $200 \mathrm{kV}$, with a resolution close to $0.7 \AA$ and a defocus value of $10 \mathrm{~nm}$; the inner and outer detector angles were set to 50 and $200 \mathrm{mrad}$ respectively, and convergence semi-angle to $24 \mathrm{mrad}$. The comparison between the real and the simulated micrograph is shown in Fig. 1d1. Here, we have identified the atomic position of gold (yellow spheres) and palladium (blue spheres) in the model. The region delimited by the white square contains a fragment of the simulated micrograph (the empty area in the 
simulated STEM image has been made transparent for clarity), and, apart from the obvious difference in the reference level of intensity, the elemental distribution proposed in the model reproduces the intensity profile obtained in the real micrograph adequately.

When the beam current was set to $3.75 \mathrm{nA}$, different results were obtained. Fig. 2a shows two nanoparticles, Au and Pd, with a diameter of $5.5 \mathrm{~nm}$. In this case, the beam current was suddenly increased from $6.2 \mathrm{pA}$ to $3.75 \mathrm{nA}$. Such a strong and quick increment resulted in a partial disintegration of the carbon substrate and an ordering of the initial disordered structure of the Pd nanoparticle. In Fig. 2a the Pd nanoparticle exhibits a disordered structure that crystallized after the beam irradiation (see white arrow in Fig. 2b). This could be the effect of desorption of some poisonous molecules attached to the surface, due to the strong beam irradiation. The coalescence also occurs through the formation of a well-structured neck which retains the original $f c c$ symmetry of the palladium and gold. The neck reached a diameter of $3 \mathrm{~nm}$ and a maximum length of $2 \mathrm{~nm}$ (Fig. 2c) to finally form an alloyed bimetallic nanoparticle (Fig. 2d). The intensity line profile was plotted along the final material in order to confirm the bimetallic nature of the nanoparticle ${ }^{35}$ (see Fig. 2e).

\section{Au@Pd}

When an alloy was used as the target, nanoparticles of $5 \mathrm{~nm}$ with icosahedral and decahedral morphologies were obtained with $\mathrm{Au}$ and Pd perfectly alloyed. Fig. 3a depicts the high-resolution image of the as-synthesized material and its correspondent reciprocal space diffractogram presenting a very well crystallized solid with no significant contrast change on the entire particle assuming a random distribution of the metals. The measured lattice constants were $0.23 \mathrm{~nm}$ and $0.144 \mathrm{~nm}$, as remarked in Fig. 3a corresponding to the $\{111\}$ and $\{220\}$ planes of an $f c c$ structure. An atomistic model was constructed to exemplify the
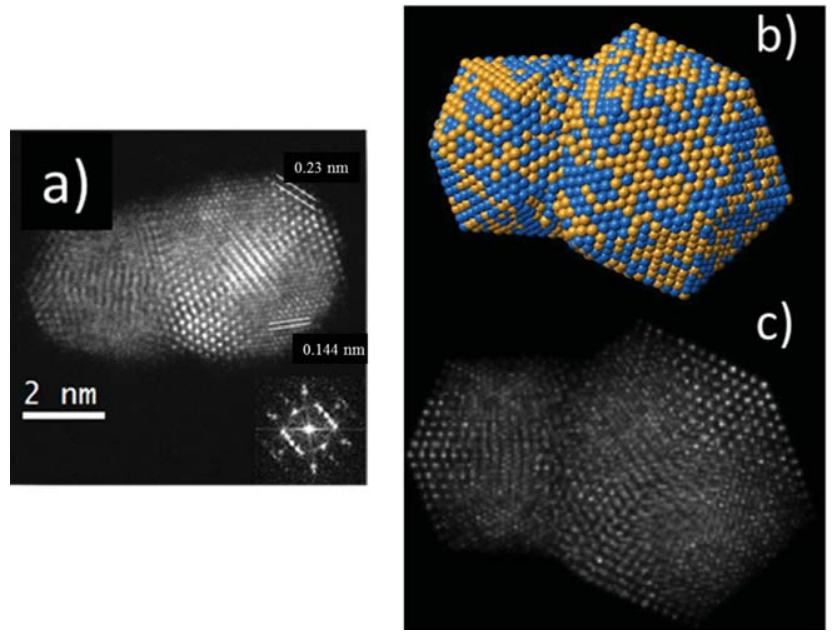

Fig. 3 (a) Cs corrected STEM-HAADF image of two icosahedral nanoparticles recorded along the [110] orientation. The two $d$-spacings marked on the image correspond to the (111) $0.144 \mathrm{~nm}$ and (200) $0.23 \mathrm{~nm}$ planes. The FFT is also shown in the inset. (b) Atomistic model representing (a) and (c) simulated STEM image of (b).

structure (Fig. 3a) which is shown in Fig. 3b where a Pd-Au nanoalloy is evident. This model was used to generate the simulated STEM micrograph shown in Fig. 3c. As observed, both the experimental and simulated images are in very good agreement.

In order to obtain a more detailed understanding of the experimental evidence, and since most previously theoretical calculations predict $\mathrm{Pd}_{\text {core }}-\mathrm{Au}_{\text {shell }}$ structures, ${ }^{20,21}$ simulated annealing calculations were performed, analyzing the stability of both naked and carbon monoxide (CO) covered $\mathrm{Au}-\mathrm{Pd}$ nanoparticles.

Following the model proposed by West et al. ${ }^{36}$ which points out the possibility of an inversion in the segregation pattern in

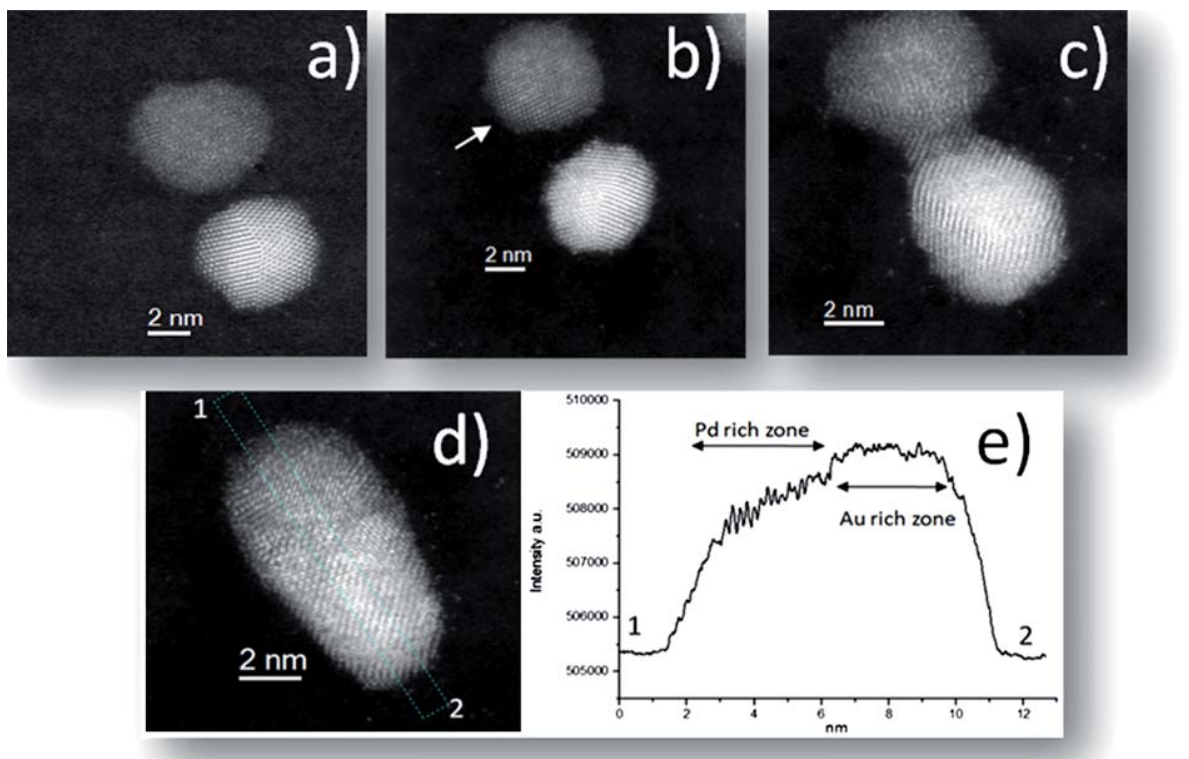

Fig. 2 (a-d) Sequence of Cs corrected STEM-HAADF images for a high electron beam irradiation (3.75 nA). (e) Intensity profile taken from (d) starting at point 1 and ending at 2 . 
the presence of $\mathrm{CO}$, from $\mathrm{Pd}_{\text {core }} \mathrm{Au}_{\text {shell }}$ to $\mathrm{Au}_{\text {core }} \mathrm{Pd}_{\text {shell }}$, we have performed atomistic simulations with a new semi-empirical potential for larger systems.

It is widely held in the nanotechnology literature that $\mathrm{CO}$ adsorption on Pd crystalline surfaces is very strong, contrary to $\mathrm{Au}$, which is known as one of the best noble metals. Even at very low $\mathrm{CO}$ concentration (i.e. $\mathrm{ppm}$ ) it is expected that most $\mathrm{CO}$ molecules present in the air will adsorb on the palladium surface. Taking this consideration into account, we have performed energetic calculations, using the simulated annealing procedure described above, in order to estimate the relative stability of different structures (bared $\mathrm{Pd}_{\text {core }} \mathrm{Au}_{\text {shell }}$, bared $\mathrm{Au}_{\text {core }} \mathrm{Pd}_{\text {shell }}$, and $\mathrm{CO}$ covered $\mathrm{Au}_{\text {core }} \mathrm{Pd}_{\text {shell }}$ ) using different coverage degree of $\mathrm{CO}$ molecules.

Fig. 4 shows the relative energy $(\Delta E)$, defined as

$$
\Delta E=\frac{\left(E_{\mathrm{Au}_{\text {core }}-\mathrm{Pd}_{\text {shell }}}^{\mathrm{NP}}-E_{\mathrm{Pd}_{\text {core }}-\mathrm{Au}_{\text {shell }}}^{\mathrm{NP}}-N_{\mathrm{CO}} E^{\mathrm{CO}}\right)}{N}
$$

where $E_{\mathrm{Au}_{\text {core }}-\mathrm{Pd}_{\text {shell }}}^{\mathrm{NP}}$ and $E_{\mathrm{Pd}_{\text {core }}}^{\mathrm{NP}}-\mathrm{Au}_{\text {shell }}$ represent the total energy of the nanoparticles with the Au core covered by Pd and vice versa; $N$ is the total number of atoms, and the last term $\left(N_{\mathrm{CO}} E^{\mathrm{CO}}\right)$ is the energy of $\mathrm{CO}$ molecules. Negative values of $\Delta E$ indicate that $\mathrm{Au}_{\text {core }} \mathrm{Pd}_{\text {shell }}$ is energetically more stable than $\mathrm{Pd}_{\text {core }} \mathrm{Au}_{\text {shell }}$.

As presented in Fig. 4, the stabilization of $\mathrm{Au}_{\text {core }} \mathrm{Pd}_{\text {shell }}$ is produced when at least $10 \mathrm{CO}$ molecules are adsorbed on a 618atom nanoparticle. Consequently, we can state that with a very small coverage of $\mathrm{CO}$ molecules ( $c a . \theta=0.04$ monolayers) a transformation from $\mathrm{Pd}_{\text {core }} \mathrm{Au}_{\text {shell }}$ to $\mathrm{Au}_{\text {core }} \mathrm{Pd}_{\text {shell }}$ is energetically stable. Some selected atomic configurations taken from the simulated annealing results are also shown in Fig. 4.

Molecular dynamics simulations in the canonical ensemble have been also performed to study the coalescence process and to

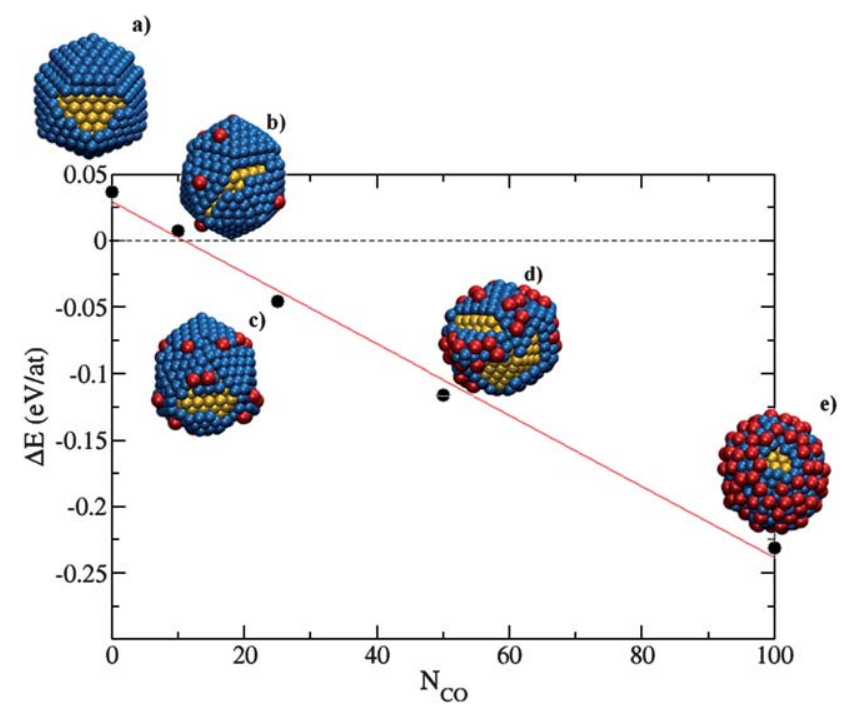

Fig. 4 Relative energy $(\Delta E)$ as a function of the number of $\mathrm{CO}$ molecules $\left(N_{\mathrm{CO}}\right)$ adsorbed on the $\mathrm{Au}_{\text {core }} \mathrm{Pd}_{\text {shell }}$ nanoparticle. Negative values of $\Delta E$ indicate that $\mathrm{Au}_{\text {core }} \mathrm{Pd}_{\text {shell }}$ is energetically more stable than $\mathrm{Pd}_{\text {core }} \mathrm{Au}_{\text {shell. }}$. The atomic configurations taken from the simulations are shown: (a) naked NP, (b) 10, (c) 25, (d) 50 and (e) $100 \mathrm{CO}$ adsorbed molecules. Yellow spheres: Au, blue spheres: Pd, and red spheres: $\mathrm{CO}$ molecules.
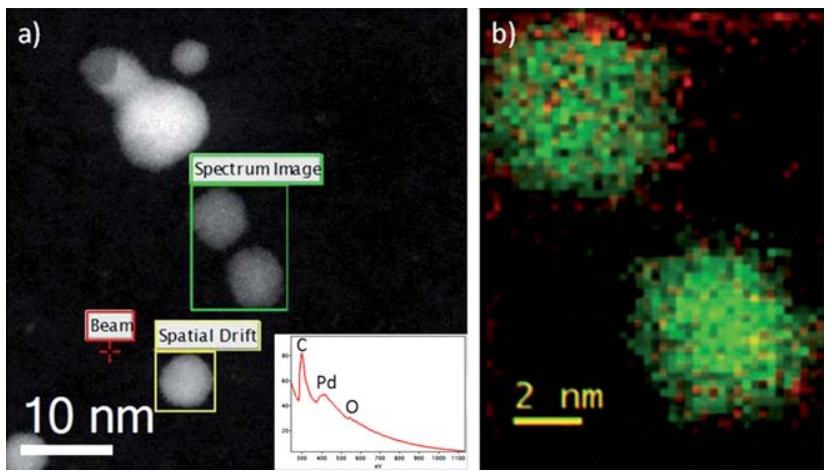

Fig. 5 Electron energy loss spectrum analysis. (a) Cs corrected STEM image where the scan analysis was taken, green rectangle named as Spectrum image. Point analysis spectrum extracted from the spectrum image is shown in inset. (b) EEL spectrum compositional map; the green color corresponds to palladium and the red color corresponds to oxygen.

compare it with the Cs-corrected STEM data. When naked particles were brought into contact, diffusion of $\mathrm{Au}$ over the $\mathrm{Pd}$ cluster was observed, in contradiction with experimental results obtained and in close agreement with all previous theoretical calculations. ${ }^{37}$ However, if $\mathrm{CO}$ molecules are adsorbed onto $\mathrm{Pd}$ nanoparticles, the diffusion of $\mathrm{Au}$ over $\mathrm{Pd}$ stops during the simulation time and Janus-like particles are observed due to the well-known simulated time limitations of the MD technique to observe infrequent events, like the ones that could be taking place in the process of CO-Pd diffusion over Au nanocrystals.

In order to experimentally corroborate the presence of oxygen within the Pd nanoparticles, electron energy loss spectroscopy (EELS) analysis was carried out. The compositional map obtained and the corresponding extracted spectrum, Fig. 5, confirm the presence of oxygen adsorbed onto the Pd nanoparticles. The green color has been attributed to palladium and the red color to oxygen. The point analysis (raw data) presented in Fig. 5a inset extracted from one of the Pd nanoparticles unambiguously confirms the presence of carbon (mostly due to the microgrid), palladium and oxygen at the same spatial position.

\section{Conclusions}

In summary, we have elucidated the alloying mechanism in small metal nanoparticles by means of state-of-the-art atomic-resolved electron microscopy. The unexpected results observed experimentally were explained with the aid of computer simulations in a virtual lab. In this sense, we propose that a very small concentration of $\mathrm{CO}$ molecules in the air should be sufficient to modify the structure of $\mathrm{Pd}$ nanoparticles, leading finally to $\mathrm{Au}_{\text {core }} \mathrm{Pd}_{\text {shell }}$ in contrast with most of the previous calculations, where the chemical effect of $\mathrm{CO}$ was neglected.

In conclusion, we have performed a new type of nano-engineering, controlling very precisely the atomic structure and chemical ordering in real time and using new nanotechnological approaches. We believe that the present work will open the possibility to tune the chemical composition of nanocatalysts very finely. 


\section{Acknowledgements}

MMM and JAOA thank CONICET, PIP:112-200801-000983 PICT2007-340, PICT-Bicentenario2010-123 for financial support and G. Diaz Cortez for her language assistance. The group at UTSA also acknowledges the NSF PREM Grant \# DMR 0934218, Title: Oxide and Metal Nanoparticles-The Interface between life sciences and physical sciences. The authors would also like to acknowledge RCMI Center for Interdisciplinary Health Research CIHR. "The project described was supported by Award Number 2G12RR013646-11 from the National Center for Research Resources. The content is solely the responsibility of the authors and does not necessarily represent the official views of the National Center for Research Resources of the National Institutes of Health."

\section{Notes and references}

1 J. A. Rodriguez, Prog. Surf. Sci., 2006, 81, 141-189.

2 L. McEwan, M. Julius, S. Roberts and J. C. Q. Fletcher, Gold Bull., 2010, 43, 298-306.

3 D. Enache, J. Edwards, P. Landon, B. Solsona-Espriu, A. Carley, A. Herzing, M. Watanabe, C. Kiely, D. Knight and G. Hutchings, Science, 2006, 311, 362-365.

4 M. Chen, D. Kumar, C. W. Yi and D. W. Goodman, Science, 2005, 310, 291-293.

5 M. O. Nutt, J. B. Hughes and M. S. Wong, Environ. Sci. Technol., 2005, 39, 1346-1353.

6 M. O. Nutt, K. N. Heck, P. Alvarez and M. S. Wong, Appl. Catal., B, 2006, 69, 115-125.

7 M. Bonarowska, B. Burda, W. Juszczyk, J. Pielaszek, Z. Kowalczyk and Z. Karpinski, Appl. Catal., B, 2001, 35, 13-20.

8 A. M. Venezia, V. L. Parola, G. Deganello, B. Pawelec and J. L. G. Fierro, J. Catal., 2003, 215, 317-325.

9 P. Landon, P. J. Collier, A. J. Papworth, C. J. Kiely and G. J. Hutchings, Chem. Commun., 2002, 2058-2059.

10 J. H. Sinfelt, Bimetallic Catalysis: Discoveries, Concepts and Applications, Wiley, 1983.

11 D. L. Trimm and Z. I. Onsan, Catal. Rev. Sci. Eng., 2001, 43, 31-84.

12 M. Bonarowska, A. Malinowski, W. Juszczyk and Z. Karpinski, Appl. Catal., B, 2001, 30, 187-193.

13 D. Ferrer, D. A. Blom, L. F. Allard, S. Mejía, E. Pérez-Tijerina and M. José-Yacamán, J. Mater. Chem., 2008, 18, 2442-2446.

14 S. J. Mejía-Rosales, C. Fernández-Navarro, E. Pérez-Tijerina, D. A. Blom, L. F. Allard and M. José-Yacamán, J. Phys. Chem. C, 2007, 111, 1256-1260.
15 E. Pérez-Tijerina, M. Gracia Pinilla, S. Mejía-Rosales, U. OrtizMéndez, A. Torres and M. José-Yacamán, Faraday Discuss., 2008, 138, 353-362.

16 E. Pérez-Tijerina, S. Mejía-Rosales, H. Inada and M. José-Yacamán, J. Phys. Chem. C, 2010, 114, 6999-7003.

17 P. J. Ferreira, G. J. la O', Y. Shao-Horn, D. Morgan, R. Makharia, S. Kocha and H. A. Gasteiger, J. Electrochem. Soc., 2005, 152A, 2256-2271.

18 Y. Shao-Horn, W. C. Sheng, S. Chen, P. J. Ferreira, E. F. Holby and D. Morgan, Top. Catal., 2007, 46, 285-305.

19 M. A. Asoro, D. Kovar, Y. Shao-Horn, L. F. Allard and P. J. Ferreira, Nanotechnology, 2010, 21, 025701.

20 R. Ismail and R. L. Johnston, Phys. Chem. Chem. Phys., 2010, 12, $8607-8619$.

21 F. Pittaway, L. O. Paz-Borbón, R. L. Johnston, H. Arslan, R. Ferrando, C. Mottet, G. Barcaro and A. Fortunelli, J. Phys. Chem. C, 2009, 113, 9141-9152.

22 I. V. Yudanov and K. M. Neyman, Phys. Chem. Chem. Phys., 2010, 12, 5094-5100.

23 L. F. Allard, A. Borisevich, W. Deng, R. Si, M. FlytzaniStephanopoulos and S. H. Overbury, J. Electron Microsc. Tech., 2009, 58, 199.

24 D. P. O’Neal, L. R. Hirsch, N. J. Halas, J. D. Payne and J. L. West, Cancer Lett., 2004, 209, 171-176.

25 C. Loo, A. Lowery, N. Halas, J. West and R. Drezek, Nano Lett., $2005,5,709-711$.

26 K. Sattler, J. Mühlbach and E. Recknagel, Phys. Rev. Lett., 1980, 45, 821-824.

27 I. M. Goldby, B. V. Issendorff, L. Kuipers and R. E. Palmer, Rev. Sci. Instrum., 1997, 68, 3327-3334.

28 M. M. Mariscal, J. A. Olmos-Asar, C. Gutierrez-Wing, A. Mayoral and M. J. Yacaman, Phys. Chem. Chem. Phys., 2010, 12, 1178511790 .

29 J. A. Olmos-Asar, A. Rapallo and M. M. Mariscal, Phys. Chem. Chem. Phys., 2011, 13, 6500-6506.

30 P. Ordejón, E. Artacho and J. M. Soler, Phys. Rev. B: Condens. Matter, 1996, 53, R10441-R10444.

31 H. L. Abbott, A. Aumer, Y. Lei, C. Asokan, R. J. Meyer, M. Sterrer, S. Shaikhutdinov and H.-J. Freund, J. Phys. Chem. C, 2010, 114, 17099-17104.

32 F. Cleri and V. Rosato, Phys. Rev. B: Condens. Matter, 1993, 48, $22-$ 33.

$33 \mathrm{http}: / /$ www.hremresearch.com

34 K. Ishizuka, Ultramicroscopy, 2001, 90, 71-83.

35 A. Mayoral, S. Mejia-Rosales, M. M. Mariscal, E. Perez-Tijerina and M. Jose-Yacaman, Nanoscale, 2010, 2, 2647-2651.

36 P. S. West, R. L. Johnston, G. Barcaro and A. Fortunelli, J. Phys. Chem. C, 2010, 114, 19678-19686.

37 R. Ferrando, J. Jellinek and R. L. Johnston, Chem. Rev., 2008, 108, 845-910. 